DUKE-TH-98-168

\title{
Lattice Gauge Description of Colliding Nuclei
}

\author{
S. A. Bass, B. Müller, and W. Pöschl \\ Department of Physics, Duke University, Durham, NC 27708-0305, USA
}

(August 16, 2021)

\begin{abstract}
We propose a novel formalism for simultaneously describing both, the hard and soft parton dynamics in ultrarelativistic collisions of nuclei. The emission of gluons from the initially coherent parton configurations of the colliding nuclei and low- $p_{t}$ color coherence effects are treated in the framework of a YangMills transport equation on a coupled lattice-particle system. A collision term is added to the transport equation to account for the remaining intermediate and high- $p_{t}$ interactions in an infrared finite manner.
\end{abstract}

Experiments with heavy ion collisions at energies above $100 \mathrm{GeV} / \mathrm{u}$, in preparation at the Relativistic Heavy Ion Collider (RHIC) in Brookhaven and the Large Hadron Collider (LHC) at CERN, will try to establish the existence of a new phase of nuclear matter, the quark-gluon plasma [1]. One of the theoretical challenges in this context is to develop a description on the basis of quantum chromodynamics (QCD) of the processes that may lead to the formation of locally equilibrated superdense matter in these nuclear reactions.

In recent years, most theoretical attempts at developing such a description have been based on the idea that, at very high energy and for heavy nuclei, the dominant mechanism of energy deposition in the central kinematical region is the perturbative scattering of partons [2 5]. Because the interactions among gluons are stronger than those involving quarks, this mechanism predicts an abundance of gluons during the early equilibration phase [6]. This concept can be generalized into a theoretical framework, called the parton cascade model [7], which formulates thermalization as a transport process involving perturbative QCD excitations, i.e. quarks and gluons [8]. The predictions of this formalism have been extensively studied by means of numerical simulations [9, 10].

One of the problems inherent in this formulation concerns the description of the initial state. The transport equations start with the assumption of a probabilistic phase space distribution of partons, whereas in reality the states of the colliding nuclei are described by coherent parton wave functions. The incoherent parton description fails, especially, at small transverse momenta, because the QCD coupling constant diverges in naive perturbation theory. Some time ago it was proposed that the proper solution to these difficulties would be the perturbative expansion, not around the "empty" QCD vacuum, but around a mean color field describing the static color field accompanying the fast moving valence quarks of the colliding nuclei [1]]. 
Because the mean color field of a heavy nucleus locally receives contributions from the quarks contained in many different nucleons, its source can be represented as a Gaussian ensemble of color charges moving along the light cone [12]. We will, therefore, refer to this model here as the random light-cone source model (RLSM). Within this framework, the energy deposition by gluonic interactions is described as classical gluon radiation at small transverse momenta [13,14, and as gluon-gluon scattering at high transverse momenta [15]. Quantum corrections to this picture [16] predict an enhancement of the glue field of the colliding nuclei at small values of the Bjorken variable $x$. The full solution of the nonlinear classical RLSM equations for the color field of two colliding nuclei requires a lattice formulation in $2+1$ dimensions [17].

The possibility of a description of inelastic gluon processes by means of the nonlinear interactions of classical color fields has also been explored numerically in studies of collision of two Yang-Mills field wave packets on a one dimensional gauge lattice [18]. These calculations gave evidence that the interaction between localized classical gauge fields can lead to the excitation of long wavelength modes in the collision, which is reminiscent of the production of an equilibrated gluon plasma.

Here we address the question how this new insight can be incorporated into the conceptual framework of the parton cascade model. First of all, it is necessary to include a coherent color field $A_{\mu}$, in addition to the incoherent quark and gluon distributions, $q_{\mathrm{f}}(r, p)$ and $g(r, p)$. The subscript "f" here denotes the various quark flavors. We will also insist on a full $(3+1)$-dimensional representation, which will permit the study of deviations from boost invariance.

Because even the classical Yang-Mills equations do not, in general, allow for global analytic solutions [19.20], we propose to solve the RLSM equations numerically on a gauge lattice. Lattice calculations in Euclidean space-time have been shown to provide a reliable approach for the calculation of static and quasi-static properties of strongly coupled quantum field theory, in particular, QCD. For dynamical systems far off equilibrium, however, one needs to study the system in real continuous time. The lattice discretization then should only be applied to the Euclidean sub-space $\mathbf{R}^{3}$. In this case it is appropriate to choose a Hamiltonian formulation rather than a Lagrangian one. We have to emphasize that this concept is neither explicitly invariant under general gauge transformations nor Lorentz invariant. However, we believe that for the type of problems described above this method is indeed useful.

One has to select a rest frame in the space $\mathbf{R} \otimes \mathbf{R}^{3}$ which in our case probably is best chosen as the center of velocity. Further, one has to adopt a gauge. The temporal gauge in the continuum $\left(A^{0}=0\right)$ seems most appropriate here [21]. A set of equations describing the evolution of the phase space distribution of quarks and gluons in the presence of a mean color field, but in the absence of collisions, was proposed more than a decade ago by Heinz [22,23]. This non-Abelian generalization of the Vlasov equation can be considered as the continuum version of the dynamics of an ensemble of classical point particles endowed with color charge and interacting with a mean color field. The equations for this dynamical system were originally derived by Wong [24].

In the following we develop a formulation of the RLSM including the ideas of Heinz and Wong. We represent the valence quarks of the two colliding nuclei as point particles moving in the space-time continuum, and interacting with a classical gauge field defined on a spatial 
lattice but with quasi-continuous time. In principle, this idea follows the proposal of $\mathrm{Hu}$ and Müller [27] for the simulation of the effects of hard thermal loops by means of colored point particles.

At this stage we are still general enough to assume that the soft modes of the gluon fields are described through gauge fields with $\mathrm{SU}(N)$ symmetry. In the associated Liealgebra $\operatorname{LSU}(N)$ we express the Hamiltonian of the above outlined system in the continuum as

$$
\begin{aligned}
H & =\sum_{i=1}^{N_{1}} \sqrt{\left|\vec{p}_{i}\right|^{2}+m_{0}^{2}}+\sum_{i=N_{1}+1}^{N_{2}} \sqrt{\left|\vec{p}_{i}\right|^{2}+m_{0}^{2}} \\
& -2 g \int d^{3} x \operatorname{Tr}\left[\mathcal{J}_{\mu} \mathcal{A}^{\mu}\right]-\frac{1}{2} \int d^{3} x \operatorname{Tr}\left[\mathcal{F}^{\mu \nu} \mathcal{F}_{\mu \nu}\right]
\end{aligned}
$$

where $\mathcal{F}^{\mu \nu}, \mathcal{A}^{\mu}, \mathcal{J}^{\mu}, \mathcal{Q}_{i} \in \operatorname{LSU}(N)$. The curly quantities denote those in the adjoint representation, which are defined as, e.g., $\mathcal{A}^{\mu}=A_{c}^{\mu} \cdot T^{c}$ with group generators $T^{c} . g$ is the gauge coupling constant, and $\mathcal{F}^{\mu \nu}$ denotes the field strength tensor of the mean color field $\mathcal{A}_{\mu}$. The moving particles generate a color current

$$
\mathcal{J}^{\nu}(x)=\sum_{i} \mathcal{Q}_{i}(t) \frac{p_{i}^{\nu}}{\sqrt{\mid \overrightarrow{\left.p_{i}\right|^{2}+m_{0}^{2}}}} \delta\left(\vec{x}-\vec{x}_{i}(t)\right),
$$

where $t$ denotes the global time in the chosen reference frame. Denoting the space-time positions, momenta, and color charges of the particles by $x_{i}^{\mu}, p_{i}^{\mu}$ and $\mathcal{Q}_{i}^{a}$, respectively, the following equations of motion are derived from the above Hamiltonian (11):

$$
\begin{aligned}
& p_{i}^{0} \frac{d x_{i}^{\mu}}{d t}=p_{i}^{\mu} \\
& p_{i}^{0} \frac{d p_{i}^{\mu}}{d t}=2 g \operatorname{Tr}\left(\mathcal{Q}_{i} \mathcal{F}^{\mu \nu}\right) p_{i, \nu} \\
& p_{i}^{0} \frac{d \mathcal{Q}_{i}}{d t}=\imath g\left[\mathcal{Q}_{i}, \mathcal{A}^{\mu}\right]_{-} p_{\mu, i}
\end{aligned}
$$

The factors $p_{i}^{0}$ on the l.h.s. are needed to convert the derivatives with respect to proper time into coordinate time derivatives. Furthermore, the inhomogeneous Yang-Mills equations

$$
D_{\mu} \mathcal{F}^{\mu \nu}(x)=g \mathcal{J}^{\nu}(x)
$$

describe the dynamics of the classical mean color fields. The current density (2) forms the source term on the r.h.s. of (6). The coupled system of the Wong equations (3-5) and the Yang-Mills equation (可) is highly nonlinear and can only be solved numerically or perturbatively.

These equations have been used to simulate the effects of hard thermal loops [26] on the dynamics of soft modes of a non-Abelian $\mathrm{SU}(2)$ gauge field at finite temperature 27.28. In

\footnotetext{
${ }^{1}$ The numerical implementation also requires a discretization of the time variable, but the temporal step size can be taken arbitrarily small.
} 
this case, the colored particles describe the gauge field modes with thermal momenta, and the mean field describes the coherent motion of those gauge field modes which have a wave number $k$ much smaller than the temperature $T$ and are highly occupied. The separation of the two regimes was achieved by discretizing the mean gauge field on a lattice with elementary spacing $a \ll T^{-1}$. Requiring particles to have momenta $p>\pi / a$ then avoids double counting degrees of freedom.

Here we propose to use the equations (2-6) to describe the interactions among the glue field components of two colliding heavy nuclei. In this case, the lattice cut-off $a$ can be used to separate the regime in transverse momentum where the dynamics of gluons is perturbative (large $k_{\mathrm{T}}$ ) from that where naive perturbation theory fails (small $k_{\mathrm{T}}$ ). The gluon propagators used for the calculation of the collision terms will be regulated in the infrared by the lattice cut-off $k_{\mathrm{c}}=\pi / a$. The interaction with the mean color field allows for an exchange of an arbitrary number of gluons, and the screening of the soft components of the gauge field by perturbative partons [29, 30] is taken into account naturally by the nonlinear nature of the coupled equations (2 6).

Following the idea of Kogut [21, we approximate the gluonic part of the Hamiltonian (1) by a discretized form on a gauge lattice. In contrast to Ref. [21], however, we represent the fermions through point-like particles. This leads to a Hamiltonian which is represented as a sum of the following terms

$$
H=H_{\mathrm{part}}+H_{\mathrm{YM}}^{(\text {lattice })}
$$

where $H_{\text {part }}$ contains the first two terms on the r.h.s. of (1) and $H_{\mathrm{YM}}^{(\text {lattice) }}$ is defined as

$$
\begin{aligned}
H_{\mathrm{YM}}^{(\text {lattice })}= & -a^{3} \sum_{x, k} \operatorname{Tr}\left\{\mathcal{E}_{x, k} \mathcal{E}_{x, k}\right. \\
& -\left(\frac{1}{4 i g a^{2}} \sum_{l, m} \varepsilon_{k l m}\left(\mathcal{U}_{x, m l}-\mathcal{U}_{x, l m}\right)\right)^{2} \\
& \left.-g \mathcal{J}_{x, k} \mathcal{A}_{x, k}\right\} .
\end{aligned}
$$

As already mentioned, the dynamical equations (2-6) can be solved efficiently by numerical time integration. A lattice version of the continuum equations is constructed [27,28] by expressing the gauge fields in terms of link variables $\mathcal{U}_{x, l} \in \mathrm{SU}(N)$, which represent the parallel transport of a field amplitude from a site $x$ to a neighboring site $(x+l)$ in the direction $l$. As in the Kogut-Susskind model [21] we choose the temporal gauge $A_{0}=0$ and define the following variables:

$$
\begin{aligned}
\mathcal{U}_{x, l} & =\exp \left(-i g a \mathcal{A}_{l}(x)\right)=\mathcal{U}_{x+l,-l}^{\dagger} \\
\mathcal{U}_{x, k l} & =\mathcal{U}_{x, k} \mathcal{U}_{x+k, l} \mathcal{U}_{x+k+l,-k} \mathcal{U}_{x+l,-l}
\end{aligned}
$$

Consequently, we have

$$
\begin{aligned}
\mathcal{E}_{x, j} & =\frac{1}{i g a} \dot{\mathcal{U}}_{x, j} \mathcal{U}_{x, j}^{\dagger} \\
\mathcal{B}_{x, j} & =\frac{1}{4 i g a^{2}} \epsilon_{j k l}\left(\mathcal{U}_{x, k l}^{\dagger}-\mathcal{U}_{x, k l}\right)
\end{aligned}
$$


for the electric and magnetic fields $\left(\mathcal{E}_{x, j}, \mathcal{B}_{x, j} \in \operatorname{LSU}(N)\right)$, respectively. There are advantages in choosing $\mathcal{U}_{x, i}$ and $\mathcal{E}_{x, i}$ as the basic dynamic field variables. This choice transforms the discretized Yang-Mills equations into the following equations of motion

$$
\begin{aligned}
\dot{\mathcal{U}}_{x, k}(t) & =i g a \mathcal{E}_{x, k}(t) \mathcal{U}_{x, k}(t) \\
\dot{\mathcal{E}}_{x, k}(t) & =\frac{1}{2 i g a^{3}} \sum_{l=1}^{3}\left\{\mathcal{U}_{x, k l}^{\dagger}(t)-\mathcal{U}_{x, k l}(t)\right. \\
& -\mathcal{U}_{x-l, l}^{\dagger}(t) \mathcal{U}_{x-l, k l}^{\dagger}(t) \mathcal{U}_{x-l, l}(t) \\
& \left.+\mathcal{U}_{x-l, l}^{\dagger}(t) \mathcal{U}_{x-l, k l}(t) \mathcal{U}_{x-l, l}(t)\right\} \\
& -g \mathcal{J}_{x, k}(t)
\end{aligned}
$$

In the spirit of the statistical nature of the transport theory, we split each quark into a number $n_{q}$ of test particles, each of which carries the fraction $q_{0}=Q_{0} / n_{q}$ of the quark color charge $Q_{0}$. In a first step, we adopt the gauge group $\mathrm{SU}(2)$ here for simplicity. Consequently, each nucleon is represented by two quarks (instead of three), initially carrying opposite color charge.

Perturbative short range interactions at high momenta can be described in form of a stochastic collision term, well known from Boltzmann-type transport equations [31, 32]. For a consistent description of both, long range and short range interactions on an equal footing, the equations of motion (3 - 5) for the long range interactions have to be cast into the form of a single transport equation and combined with the collision term. The Vlasov part of the transport equation, from which the equations of motion (3 - 5) can be recovered, was first derived in 22, 23. We extend the formulation by adding a stochastic collision term similar to the one used in [7]. The full transport equation then follows as:

$$
\begin{aligned}
p_{i}^{0} \frac{\mathrm{df}_{k}\left(x_{i}^{\mu}, p_{i}^{\mu}, \mathcal{Q}_{i}\right)}{\mathrm{d} t} \equiv & p_{i}^{\mu}\left\{\partial_{\mu}-2 g \operatorname{Tr}\left(\mathcal{Q}_{i} \mathcal{F}^{\mu \nu}\right) \partial_{p}^{\nu}\right. \\
& \left.+2 \imath g \operatorname{Tr}\left(\left[\mathcal{Q}_{i}, \mathcal{A}^{\mu}\right]_{-} \partial_{\mathcal{Q}}\right)\right\} \mathrm{f}_{k}\left(x_{i}^{\mu}, p_{i}^{\mu}, \mathcal{Q}_{i}\right) \\
= & \sum_{\text {processes }} C\left(p_{i}^{\mu}, x_{i}^{\mu}, \mathcal{Q}_{i}, t\right) .
\end{aligned}
$$

Here $\mathrm{f}_{k}$ denotes the one-particle distribution functions of the valence quarks and of the "hard" gluons $(k=\mathrm{q}, \mathrm{g})$. This set of nonlinear integro-differential equations is coupled to the Yang-Mills equation in which the color current is now given by a moment of the one-particle distribution functions:

$$
D_{\mu} \mathcal{F}^{\mu \nu}(x)=g \sum_{k} \int \mathrm{d} \mathcal{Q}_{i} \frac{\mathrm{d}^{3} p_{i}}{p_{i}^{0}} \mathcal{Q}_{i} p_{i}^{\nu} \mathrm{f}_{k}\left(p_{i}^{\nu}, x_{i}^{\nu}, \mathcal{Q}_{i}\right)
$$

The collision integrals have the form:

$$
\begin{aligned}
C\left(p_{i}^{\mu}, x_{i}^{\mu}, \mathcal{Q}_{i}, \tau\right)= & \frac{1}{2 S_{i}} \cdot \int \theta\left(\left|p_{i}\right|-\left|k_{c}\right|\right) \prod_{j} \mathrm{~d} \Gamma_{j}\left|\mathcal{M}^{(c)}\right|^{2} \\
& (2 \pi)^{4} \delta^{4}\left(P_{\text {in }}-P_{\text {out }}\right) D\left(\mathrm{f}_{k}\left(p_{i}^{\mu}, x_{i}^{\mu}, \mathcal{Q}_{i}\right)\right)
\end{aligned}
$$

with 


$$
D\left(\mathrm{f}_{k}\left(p_{i}^{\nu}, x_{i}^{\nu}, \mathcal{Q}_{i}\right)\right)=\prod_{\text {in }} \mathrm{f}_{k}\left(p_{i}^{\nu}, x_{i}^{\nu}, \mathcal{Q}_{i}\right)-\prod_{\text {out }} \mathrm{f}_{k}\left(p_{i}^{\nu}, x_{i}^{\nu}, \mathcal{Q}_{i}\right)
$$

and

$$
\prod_{j} \mathrm{~d} \Gamma_{j}=\prod_{\substack{j \neq i \\ \text { in,out }}} \frac{\mathrm{d}^{3} p_{j}}{\left(2 \pi^{3}\right)\left(2 p_{j}^{0}\right)} \theta\left(\left|p_{j}\right|-\left|k_{c}\right|\right) .
$$

$S_{i}$ is a statistical factor defined as

$$
S_{i}=\prod_{j \neq i} K_{a}^{\mathrm{in} !} K_{a}^{\mathrm{out} !}
$$

with $K_{a}^{\text {in,out }}$ identical partons of species $a$ in the initial or final state of the process, excluding the $i$ th parton.

The step functions $\theta\left(\left|p_{i}\right|-\left|k_{c}\right|\right)$ insure that only hard particles are allowed to propagate in the system. The superscript $(c)$ on the matrix element $\mathcal{M}$ indicates that only the hard, i.e. short range, part of the interaction is treated in the collision term. This cut-off will be discussed in more detail below.

The matrix elements $\left|\mathcal{M}^{(c)}\right|^{2}$ account for the following processes:

$$
\begin{aligned}
& \text { A } \quad q+q^{\prime} \rightarrow q+q^{\prime}, \\
& \text { B } q+q \rightarrow q+q \text {, } \\
& \mathrm{C} \quad q+\bar{q} \rightarrow g+g \text {, } \\
& \text { D } \quad g+g \rightarrow g+g \text {, }
\end{aligned}
$$

together with those obtained from crossing relations ( $q$ and $q^{\prime}$ denote different quark flavors). The amplitudes for these processes - not taking the infrared lattice cut-off $k_{c}$ into account - have been calculated in refs. [33 35 for massless quarks and in refs. 36, 37 for massive quarks. The corresponding scattering cross sections are expressed in terms of spin- and color-averaged amplitudes $\left|\mathcal{M}^{(c)}(\hat{s}, \hat{t}, \hat{u})\right|^{2}$ :

$$
\frac{\mathrm{d} \hat{\sigma}^{(\mathrm{A}, \mathrm{B}, \mathrm{C}, \mathrm{D})}(\hat{s}, \hat{t}, \hat{u})}{\mathrm{d} \hat{t}}=\frac{1}{16 \pi \hat{s}^{2}}\left\langle\left|\mathcal{M}^{(c)}(\hat{s}, \hat{t}, \hat{u})\right|^{2}\right\rangle
$$

with $\hat{s}, \hat{t}, \hat{u}$ being the well-known Mandelstam variables. For the transport calculation we also need the total cross section as a function of $\hat{s}$ which can be obtained from (22):

$$
\hat{\sigma}_{a b}(\hat{s})=\sum_{c, d} \int_{\hat{t}_{\min }}^{\hat{t}_{\text {max }}}\left(\frac{\mathrm{d} \hat{\sigma}\left(\hat{s}, \hat{t}^{\prime}, \hat{u}\right)}{\mathrm{d} \hat{t}^{\prime}}\right)_{a b \rightarrow c d} \mathrm{~d} \hat{t}^{\prime} .
$$

The integration boundaries are fixed through kinematical constraints. Note that the treatment of the cross section (21 23) with the matrix elements supplied in [33 37] does not take the infrared lattice cut-off $k_{c}$ into account. The rigorous way to evaluate the matrix elements $\left|\mathcal{M}^{(c)}\right|^{2}$ and to eliminate the small momenta from the gluon propagators would be to subtract the lattice propagator from the continuum propagator in the Feynman diagram describing the scattering process at lowest order. Because the evaluation of the gluon propagator on 
the lattice is complicated, we propose here to use, for exploratory studies, the usual matrix elements [33] 37] but with a cut-off on the allowed momentum transfer, corresponding to the lattice cut-off $k_{c}=\pi / a$. We can cast this into the Lorentz invariant form that the scale of the interaction, $Q^{2}(\hat{s}, \hat{t}, \hat{u})$ must satisfy the constraint

$$
Q^{2}(\hat{s}, \hat{t}, \hat{u})>k_{c}^{2}
$$

The functional form of $Q^{2}$ is generally process dependent and not unambiguous, although at leading order all choices for $Q$ that increase with the parton-parton center-of-mass energy are equivalent. One can now solve equation (24) for $\hat{t}$ in order to obtain an additional constraint for the integration boundaries of equation (23). Thus, only momentum transfers larger than $k_{c}$ contribute to the total cross section. It was shown in ref. [15] that spectrum of the classical Yang-Mills radiation matches smoothly onto the conventional minijet distribution near the intrinsic transverse momentum scale of the partons in a heavy nucleus at high energy. The resulting expectation that the precise choice of the momentum cutoff $k_{c}$ is not important must, of course, be verified by future numerical calculations.

In summary, we have developed a novel formalism, which allows for the first time the treatment of both, the hard and the soft parton dynamics in ultrarelativistic heavy ion collisions in a consistent transport approach: The emission of gluons from the initially coherent parton configurations of the colliding nuclei as well as low- $p_{t}$ color coherence effects in parton-parton scatterings are treated in the framework of a Yang-Mills transport equation on a coupled lattice-particle system. Intermediate and high- $p_{T}$ interactions are described in a collision term similar to that of the parton cascade model. This formalism thus avoids problems connected to the infrared cut-offs in the parton cascade model and offers a unified treatment of coherence effects within that approach.

Acknowledgments: We gratefully acknowledge remarks from Ulrich Heinz which helped to improve our manuscript. One of us (S.A.B.) acknowledges support from a Feodor Lynen Fellowship of the Alexander v. Humboldt Foundation. This work was supported in part by a grant from the U.S. Department of Energy DE-FG02-96ER40495. 


\section{REFERENCES}

[1] J. W. Harris and B. Müller, Annu. Rev. Nucl. Part. Sci. 46, 71 (1996)

[2] R.C. Hwa and K. Kajantie, Phys. Rev. Lett. 56, 696 (1986)

[3] J.P. Blaizot and A.H. Mueller, Nucl. Phys. B289, 847 (1987)

[4] K.J. Eskola, K. Kajantie, and J. Lindfors, Phys. Lett. B214, 613 (1988); Nucl. Phys. B323, 37 (1989)

[5] M. Gyulassy and X.N. Wang, Phys. Rev. D44, 3501 (1991)

[6] E.V. Shuryak, Phys. Rev. Lett. 68, 3270 (1992)

[7] K. Geiger and B. Müller, Nucl. Phys. B369, 600 (1992)

[8] K. Geiger, Phys. Rev. D56, 2665 (1997)

[9] K. Geiger, Phys. Rep. 258, 378 (1995)

[10] K. Geiger, Comp. Phys. Comm. 104, 70 (1997)

[11] L. McLerran and R. Venugopalan, Phys. Rev. D49, 2233 and 3352 (1994)

[12] Yu.V. Kovchegov, Phys. Rev. D54, 5463 (1996); D55, 5455 (1997)

[13] A. Kovner, L. McLerran, and H. Weigert, Phys. Rev. D52, 3809 and 6231 (1995)

[14] Yu.V. Kovchegov and D.H. Rischke, Phys. Rev. C56, 1084 (1997)

[15] M. Gyulassy and L. McLerran, Phys. Rev. C56, 2219 (1997)

[16] J. Jalilian-Marian, A. Kovner, L. McLerran, and H. Weigert, Phys. Rev. D55, 5414 (1997); J. Jalilian-Marian, A. Kovner, A. Leonidov, and H. Weigert, Nucl. Phys. B504, 415 (1997)

[17] A. Krasnitz and R. Venugopalan, preprint NBI-HE-97-26 (hep-ph/9706329)

[18] C.R. Hu, S.G. Matinyan, B. Müller, and A. Trayanov, Phys. Rev.D 52, 2402 (1995)

[19] S.G. Matinyan, G.K. Savvidy, N.G. Ter-Arutyunyan-Savvidy, Sov. Phys. JETP 53, 412 (1981); JETP Lett. 34, 590 (1981)

[20] J. Froyland, Phys. Rev. D27, 943 (1983); Phys. Rev. Lett. 51, 351 (1983)

[21] J. Kogut and L. Susskind, Phys. Rev. D11, 395 (1975)

[22] U. Heinz, Phys. Rev. Lett. 51, 351 (1983); Ann. Phys. (NY) 161, 48 (1985); 168, 148 (1986)

[23] H.-Th. Elze and U. Heinz, Phys. Rep. 183, 81 (1989)

[24] S.K. Wong, Nuovo Cim. A 65, 689 (1979)

[25] U. Heinz, Phys. Lett. B 144, 228 (1984)

[26] R. Pisarski, Phys. Rev. Lett. 63, 1129 (1989); E. Braaten and R. Pisarski, Phys. Rev. D 42, 2156 (1990)

[27] C.R. Hu and B. Müller, Phys. Lett. B409, 377 (1997)

[28] G.D. Moore, C.R. Hu and B. Müller, Phys. Rev. D58, 045001 (1998)

[29] T.S. Biró, B. Müller, and X.N. Wang, Phys. Lett. B283, 171 (1992)

[30] K.J. Eskola, B. Müller, and X.N. Wang, Phys. Lett. B374, 20 (1996)

[31] G. Bertsch, S. Das Gupta and H. Kruse, Phys. Rev. C29, 673 (1984)

[32] H. Kruse, B.V. Jacak and H. Stöcker, Phys. Rev. C31, 1770 (1985)

[33] R. Cutler and D. Sivers, Phys. Rev. D17, 196 (1978)

[34] B.L. Combridge, J. Kripfganz and J. Ranft, Phys. Lett. B70, 234 (1977)

[35] H.U. Bengtsson, Comp. Phys. Com 31, 323 (1984).

[36] B.L. Combridge, Nucl. Phys. B151, 429 (1979)

[37] P. Nason, S. Dawson and R.K. Ellis, Nucl. Phys. B303, 607 (1988); B327, 49 (1989); B335, 260 (1990) 